\title{
Oculocardiac Reflex
}

National Cancer Institute

\section{Source}

National Cancer Institute. Oculocardiac Reflex. NCI Thesaurus. Code C118863.

Slowing or cessation of the heart rate secondary to traction on the extraocular muscle. 